\title{
Telework and telerehabilitation programs for workers with a stroke during the COVID-19 pandemic: A commentary
}

\author{
Vahideh Moradi ${ }^{\mathrm{a}, \mathrm{b}}$, Taher Babaee ${ }^{\mathrm{a}, *}$, Elham Esfandiari ${ }^{\mathrm{c}}$, Shannon B. Lim ${ }^{\mathrm{c}}$ and Ramin Kordi ${ }^{\mathrm{b}}$ \\ ${ }^{a}$ Rehabilitation Research Center, Department of Orthotics and Prosthetics, School of Rehabilitation Sciences, \\ Iran University of Medical Sciences, Tehran, Iran \\ ${ }^{\mathrm{b}}$ Sports Medicine Research Center, Tehran University of Medical Sciences, Tehran, Iran \\ ${ }^{\mathrm{c}}$ Graduate Program in Rehabilitation Sciences, University of British Columbia, Vancouver, BC, Canada
}

Received 6 October 2020

Accepted 25 October 2020

\begin{abstract}
.
BACKGROUND: Due to the coronavirus disease 2019 (COVID-19) pandemic, rehabilitation facilities have become less accessible for patients with a stroke. Lack of early, intensive rehabilitation misses the opportunity for recovery during the critical time window of endogenous plasticity and improvement post-stroke.

OBJECTIVE: The purpose of this commentary was to highlighting the benefits of telework and telerehabilitation programs for workers with a stroke during the COVID-19 pandemic.

METHODS: Relevant publications regarding the management of individuals with a stroke, telerehabilitation and teleworking in the setting of COVID-19 were reviewed.

RESULTS: Previous studies showed that telerehabilitation can effectively provide an alternate method of promoting recovery for patients with a stroke. With the physical distancing precautions in place for mitigating viral spread, teleworking can also provide a method for long term recovery and improvements in quality of life after a stroke.

CONCLUSIONS: Overall, this commentary addresses the benefits of physically distant, safe and effective alternatives to support individuals who live with a stroke during COVID-19 pandemic.
\end{abstract}

Keywords: Rehabilitation, telehealth, work, return to work

\section{Introduction}

The coronavirus disease 2019 (COVID-19) has caused an unprecedented crisis in all social, economic and health areas $[1,2]$. The immediate response has been physical distancing (i.e., limiting social gatherings, restricting public transportation systems,

\footnotetext{
*Address for correspondence: Dr. Taher Babaee, Rehabilitation Research Center, Department of Orthotics and Prosthetics, School of Rehabilitation Sciences, Iran University of Medical Sciences, Tehran, Iran. Tel.: +98 212222 8051; E-mail: babaee.t@iums.ac.ir.
}

restricting certain businesses, etc.) as an attempt to slow down the spread of virus [3]. Healthcare facilities have been restricted to more urgent cases, and most rehabilitation treatments, especially those related to chronic conditions, have been suspended to try and protect the most vulnerable patients. However, the continuation of rehabilitation programs for certain chronic conditions, such as stroke, is vital for recovery of patients [4]. Indeed, under the current physical distancing precautions, a large number of patients cannot benefit from appropriate services and many of their treatments have unfortunately been 
suspended. Given the unprecedented nature of this pandemic, and the uncertain timing of the mandatory suspension of important rehabilitation services, there are two questions we have raised: First, what is the effect of COVID-19 pandemic on individuals with a stroke? Second, what solutions are available to promote recovery within this population?

\section{Effects of COVID-19 on individuals with stroke}

Stroke is one of the most common causes of disability and mortality, worldwide [5]. According to the World Health Organization (WHO), 15 million people suffer from a stroke annually, of which five million are left permanently debilitated [6]. Stroke is associated with depression and physical disability, which limit Activities of Daily Living (ADLs), social engagement, and overall decreased quality of life $[7,8]$. Stroke causes an economic burden at both the individual and societal level [9]. The treatment programs after stroke are an intensive and indefinite course of therapy, and include a variety of rehabilitation programs, including occupational therapy, physiotherapy, orthotic treatment, and speech and language therapy $[10,11]$. These programs are typically intensively administered early post-stroke to optimize the window of endogenous plasticity and recovery of impairment and function [12]. It is also important to consider the significant effect of depression that not uncommonly occurs secondary to a stroke; depression has been shown to reduce an individual's motivation to follow their rehabilitation programs [7]. As such, a therapists' supervision and follow-up are vital in achieving successful treatment programs following a stroke. These intensive programs and ongoing treatments can increase the chance of returning to their vocation post-stroke [13].

Unfortunately, many rehabilitation facilities have suspended treatment programs for patients with a stroke to reduce the risk of COVID-19 transmission. Suspension of such services are likely detrimental to overall health post-stroke; in fact, Zhou et al. [14] reported a higher mortality rate in people with comorbidity such as stroke, after being infected by COVID-19. Given the importance of rehabilitation programs in recovery of individuals with a stroke, physical distancing has caused the loss of recovery both intensively during the optimal time window and ongoing with guidance. Additionally, the fear of being infected with COVID-19 has exacerbated depression and stress in vulnerable patients with a stroke [15]. With all these in consideration, COVID19 isolation is associated with physical and mental consequences for patients with a stroke, such as depression, motor dysfunction, decreased physical activity and a delay in returning to work [16].

\section{Potential solutions}

Telerehabilitation can help to provide the support and care for patients with a stroke while reducing the risk of COVID-19 transmission [17]. Telerehabilitation is the use of technology to communicate between the patient and the therapist, and deliver the rehabilitation programs at a distance [18]. A randomized clinical trial conducted by Cramer et al. [19] indicated that telerehabilitation as usual rehabilitation is an effective strategy in improving arm motor function for adults with a stroke. Also, Øra et al. [20] investigated the feasibility and acceptability of speech and language therapy by videoconference in individuals with aphasia following stroke, which resulted in improvement in language function. Telerehabilitation not only lead to improvement of the quality of life in individuals with stroke, but also decreased depression in their caregivers [21]. There are, however, challenges associated with using technology in the delivery of health programs to patients with a stroke, especially older adults, such as lack of knowledge and education and confidence towards using technology [22]. Despite this, adoption of alternative methods for delivering health programs is critical to allow monitoring, guidance, and support by healthcare professionals. Given the majority of people with a stroke are adults aged greater than 65 years old, telephone screening can potentially augment the effect of telehealth programs positively. Telephone is the most common delivery method for telerehabilitation programs for older adults [23]; it could therefore be used to establish the treatment plan for patients with a stroke [24]. Furthermore, family members or caregivers can help the patients with a stroke to receive the online healthcare programs.

\section{Teleworking}

Telework is a flexible way of working at a remote location that was suggested to prevent COVID-19 transmission. Unfortunately, telework has several challenges, such as lack of motivation to work, and issues with ergonomics [25]. Of course, this is not 
unique to patients who suffer from a stroke, but is likely to be one of many things that is more difficult to perform in comparison to the person without disability [26]. Thankfully, telework can be helpful for patients with physical disability secondary to a stroke. This is important, as returning to work is associated with increased self-esteem and quality of life in patients with a stroke [27]. However, about half of the individuals with a stroke do not return to work [28]. This is not surprising as most jobs require good physical condition, adequate speech and language function, attention, etc. Based on a previous systematic review, functional dependency and cognitive ability are the most common factors influencing return to work [16]. Returning to work for someone using an assistive device such as a brace or wheelchair is challenging and the extra physical task of commuting to work is fatiguing [29]. Telework can be a potential solution to decrease the disruptions in daily work life for patients with a stroke by overcoming the inherent barriers that follow such a difficult experience. Using telework, the individual will be able to balance between their fatigue and working hours while achieving maximum productivity. In addition, telework can reduce psychological stress associated with unpredictable frustration and impaired cognitions.

\section{Conclusion}

Rehabilitation programs following a stroke can facilitate returning to work and in turn improve overall quality of life. In the midst of a pandemic, alternative methods of providing rehabilitation and ongoing work are needed to optimize overall recovery in a safe and effective manner. Telerehabilitation and telework are the potential solutions to effectively deliver this need while reducing the COVID-19 transmission, cost [30] and geographical boundaries of inaccessible rehabilitation facilities for patients with a stroke. While some work supports the use of tele-modalities, additional research is needed to further determine barriers associated with distance rehab/work, the efficacy of specific telerehabilitation interventions, and effective implementation of telerehabilitation programs.

\section{Conflict of interest}

The authors declare no potential conflicts of interests with respect to the research, authorship, and/or publication of this article.

\section{References}

[1] Soheili S, Shariat A, Anastasio A. Modification of existing occupational therapeutic protocols in response to new normal life after COVID 19: Letter to the Editor. Work. 2020 (Preprint):1-2.

[2] Memari A, Shariat A, Anastasio A, Hakakzadeh A. Rising incidence of musculoskeletal discomfort in the wake of the COVID-19 crisis. Work. (Preprint):1-3.

[3] Bedford J, Enria D, Giesecke J, Heymann DL, Ihekweazu C, Kobinger G, et al. COVID-19: towards controlling of a pandemic. The Lancet. 2020;395(10229):1015-8.

[4] Bernhardt J, Godecke E, Johnson L, Langhorne P. Early rehabilitation after stroke. Curr Opin Neurol. 2017;30(1): 48-54.

[5] Mukherjee D, Patil CG. Epidemiology and the global burden of stroke. World neurosurg. 2011;76(6):S85-S90.

[6] Mackay J, Mensah GA. The atlas of heart disease and stroke. World Health Organization; 2004.

[7] Carod-Artal FJ, Egido JA. Quality of life after stroke: the importance of a good recovery. Cerebrovasc Dis. 2009;27(Suppl. 1):204-14.

[8] Di Carlo A. Human and economic burden of stroke. Oxford University Press; 2009.

[9] Brox J, Storheim K, Grotle M, Tveito T, Indahl A, Eriksen $\mathrm{H}$. Systematic review of back schools, brief education, and fear-avoidance training for chronic low back pain. Spine J. 2008;8(6):948-58.

[10] Shariat A, Nakhostin Ansari N, Honarpishe R, Moradi V, Hakakzadeh A, Cleland JA, et al. Effect of cycling and functional electrical stimulation with linear and interval patterns of timing on gait parameters in patients after stroke: a randomized clinical trial. Disabil Rehabil. 2019:1-7.

[11] Brady MC, Godwin J, Enderby P, Kelly H, Campbell P. Speech and language therapy for aphasia after stroke: An updated systematic review and meta-analyses. Stroke. 2016;47(10):e236-e237.

[12] Bernhardt J, Hayward KS, Kwakkel G, Ward NS, Wolf SL, Borschmann K, et al. Agreed definitions and a shared vision for new standards in stroke recovery research: the stroke recovery and rehabilitation roundtable taskforce. Int J Stroke. 2017;12(5):444-50.

[13] Wei X-j, Liu X-f, Fong KN. Outcomes of return-to-work after stroke rehabilitation: A systematic review. Br J Occup Ther. 2016;79(5):299-308.

[14] Zhou F, Yu T, Du R, Fan G, Liu Y, Liu Z, et al. Clinical course and risk factors for mortality of adult inpatients with COVID-19 in Wuhan, China: a retrospective cohort study. The lancet. 2020;395(10229):1054-62.

[15] Sylaja P, Srivastava MP, Shah S, Bhatia R, Khurana D, Sharma A, et al. The SARS-CoV-2/COVID-19 pandemic and challenges in stroke care in India. Ann N Y Acad Sci. 2020 May 12 [Epub ahead of print]. doi: 10.1111/nyas. 14379

[16] Edwards JD, Kapoor A, Linkewich E, Swartz RH. Return to work after young stroke: a systematic review. Int J Stroke. 2018;13(3):243-56.

[17] Sarfo FS, Ulasavets U, Opare-Sem OK, Ovbiagele B. Telerehabilitation after stroke: an updated systematic review of the literature. J Stroke Cerebrovasc Dis. 2018;27(9):230618.

[18] Shariat A, Hakakzadeh A, Cleland J. Home-based exercise note in Covid-19 quarantine situation for office workers: A commentary. Work. 2020 (Preprint):1-2. 
[19] Cramer SC, Dodakian L, Le V, See J, Augsburger R, McKenzie A, et al. Efficacy of home-based telerehabilitation vs in-clinic therapy for adults after stroke: a randomized clinical trial. JAMA Neurology. 2019;76(9):1079-87.

[20] Øra HP, Kirmess M, Brady MC, Sørli H, Becker F. Technical Features, Feasibility, and Acceptability of Augmented Telerehabilitation in Post-stroke Aphasia-Experiences From a Randomized Controlled Trial. Front Neurol. 2020;11:671.

[21] Smith GC, Egbert N, Dellman-Jenkins M, Nanna K, Palmieri PA. Reducing depression in stroke survivors and their informal caregivers: A randomized clinical trial of a web-based intervention. Rehabil Psychol. 2012;57(3):196.

[22] Engström A-LL, Lexell J, Lund ML. Difficulties in using everyday technology after acquired brain injury: a qualitative analysis. Scand J Occup Ther. 2010;17(3):233-43.

[23] Lemke M, Rodríguez Ramírez E, Robinson B, Signal N. Motivators and barriers to using information and communication technology in everyday life following stroke: a qualitative and video observation study. Disabil Rehabil. 2020;42(14):1954-62.

[24] Kamoen O, Maqueda V, Yperzeele L, Pottel H, Cras P, Vanhooren G, et al. Stroke coach: a pilot study of a personal digital coaching program for patients after ischemic stroke. Acta Neurol Belg. 2020;120(1):91-7.
[25] de Macêdo TAM, Cabral ELdS, Silva Castro WR, de Souza Junior CC, da Costa Junior JF, Pedrosa FM, et al. Ergonomics and telework: A systematic review. Work. 2020;66:777-88.

[26] Katsabian T. The Telework Virus: How the COVID-19 Pandemic Has Affected Telework and Exposed Its Implications for Privacy and Equality. Available at SSRN: 2020 September 1 [Epub ahead of print]. doi.org/10.2139/ssrn.3684702

[27] Treger I, Shames J, Giaquinto S, Ring H. Return to work in stroke patients. Disabil Rehabil. 2007;29(17):1397-403.

[28] Chang WH, Sohn MK, Lee J, Kim DY, Lee S-G, Shin YI, et al. Return to work after stroke: The KOSCO Study. J Rehabil Med. 2016;48(3):273-9.

[29] Andersen G, Christensen D, Kirkevold M, Johnsen S. Poststroke fatigue and return to work: a 2-year follow-up. Acta Neurol Scand. 2012;125(4):248-53.

[30] Lloréns R, Noé E, Colomer C, Alcañiz M. Effectiveness, usability, and cost-benefit of a virtual reality-based telerehabilitation program for balance recovery after stroke: A randomized controlled trial. Arch Phys Med Rehabil. 2015;96(3):418-425. e2. 